\title{
Cap layer induced stress in InAs/(AI)GaAs quantum dots
}

\author{
Shen-De Chen, Ying-Ying Chen, and Si-Chen Lee ${ }^{\text {a) }}$ \\ Department of Electrical Engineering, Graduate Institute of Electronics Engineering, National Taiwan \\ University, Taipei, Taiwan, Republic of China
}

(Received 19 May 2005; accepted 25 July 2005; published 22 September 2005)

\begin{abstract}
Photoluminescence (PL) of self-assembled InAs quantum dots (QDs) on GaAs substrate with different $\operatorname{In}(\mathrm{Al}) \mathrm{GaAs}$ cap layer are studied. It is now well known that the peak position of the InAs QDs covered with InGaAs layer shifts to longer wavelength than those covered with GaAs and AlGaAs. The theoretical simulations are developed to distinguish various contributions to the wavelength shift from the dot height, strain, and barrier height of different cap layers. It reveals that most of the stress in the InAs QDs comes from the upper GaAs cap layer. (C) 2005 American Vacuum Society. [DOI: 10.1116/1.2052710]
\end{abstract}

\section{INTRODUCTION}

Self-assembled InAs QDs grown on GaAs substrate using Stranski-Krastanov (SK) growth mode by molecular beam epitaxy (MBE) have attracted wide attention and been applied to optoelectronic devices, i.e., $1.3 \mu \mathrm{m}$ wavelength laser diode, ${ }^{1-5}$ quantum dot infrared photodetector (QDIP) ${ }^{6-10}$ But there is adversity that the vertical dimension of InAs/GaAs QDs cannot be grown higher than a certain limit, i.e., $\sim 5$ monolayer (ML) InAs, without degrading the dot quality. The PL peak position is then limited to around $1.1 \mu \mathrm{m}$ at $11 \mathrm{~K}$ and far from $1.3 \mu \mathrm{m}$ at $300 \mathrm{~K}$. One of the methods to push it to longer wavelength is to grow an InGaAs cap layer on InAs/GaAs QDs before depositing the GaAs barrier layer. ${ }^{11,12}$ It has been attributed to the strain-induced band gap shift ${ }^{13}$ and phase separation of InAs and GaAs during the growth. ${ }^{14}$ To understand the detailed mechanism of the phenomenon, both theoretical calculations and experiments were performed. All factors which may affect the PL peak energy, i.e., dot height, strain, and barrier height, etc., are carefully considered.

When depositing InAs on GaAs with thickness less than a critical thickness of 1.7 ML, it forms wetting layer whose in-plane lattice constant is compressed to be the same as that of the GaAs substrate. When the InAs layer is thicker than 1.7 ML, the build-up strain forces the InAs to start the 3D growth and forms QDs on top of the wetting layer. At the same time, the lattice constant of InAs gradually relaxes toward the unstrained bulk value, i.e., $0.6058 \mathrm{~nm}$. The stress remaining in the QDs becomes less than that of the wetting layer. The magnitude of the anisotropic stress inside the QDs depends on the amount of InAs deposited. ${ }^{15}$ Then the deposition of GaAs or $\mathrm{In}(\mathrm{Al}) \mathrm{GaAs}$ cap layer on top of InAs QDs introduces additional compressive stress, which causes blueshift of PL peak. To extract the magnitude of stress in QDs through theoretical fitting of the PL peak energy, three strained conditions for QDs were considered: (1) Fullystrained QDs: if the 3D lattice constant of InAs is forced to be the same as those of the lower GaAs and upper AlGaAs

${ }^{a)}$ Electronic mail: sclee@cc.ee.ntu.edu.tw cap layer. Since the lattice constant of AlGaAs is less than $0.2 \%$ difference from that of GaAs, they are treated as the same. (2) Unstrained QDs: if the lattice constant of InAs QDs is fully relaxed to the bulk value and not influenced by either GaAs or AlGaAs. The fully-strained and unstrained QDs do not exist in reality. (3) Partially-strained QDs: when the stress remaining in the QDs is in between the above two extreme cases. It is known that InAs/GaAs QDs grown by MBE with SK growth mode are all partially strained. ${ }^{15}$

\section{EXPERIMENTS}

We have grown four samples A, B, C, and D by solidsource molecular beam epitaxy (MBE) on semi-insulating GaAs (100) substrates using a VG Semicon V80 MBE machine. First, a $500 \mathrm{~nm}$ intrinsic GaAs layer is deposited on the substrate at $580{ }^{\circ} \mathrm{C}$, followed by the deposition of $3 \mathrm{ML}$ InAs QDs at $510^{\circ} \mathrm{C}$. After the growth of InAs QDs, all shutters were closed for $5 \mathrm{~s}$ to allow atoms to migrate on the surface. During the shutter-closed period, the arsenic atoms come from the remaining As in the MBE chamber. The growth rate of InAs QDs is $0.5 \mathrm{ML} / \mathrm{s}$. This single layer of surface InAs QDs is designated as sample A which can be used for the measurements of the PL spectra and atomic force microscopy (AFM). The sample structure is shown in Fig. 1(a). Followed by the deposition of $50 \mathrm{~nm}$ thick GaAs at $510{ }^{\circ} \mathrm{C}$ again, i.e., InAs QDs are sandwiched between lower and upper GaAs, this sample of GaAs-strained InAs QDs is designated as sample B, as shown in Fig. 1(b). These two samples were grown to investigate the origin of the stresses, i.e., whether coming mainly from upper or lower GaAs. Another two samples were grown in the same conditions, except for the different cap layers. After the 500-nm-GaAs and the 3-ML-InAs-QDs growth at 580 and $510{ }^{\circ} \mathrm{C}$, respectively, the sample $\mathrm{C}$ is followed by the deposition of $6 \mathrm{ML}$ $\mathrm{In}_{0.33} \mathrm{Ga}_{0.67} \mathrm{As}+50 \mathrm{~nm} \mathrm{GaAs}$ at $510{ }^{\circ} \mathrm{C}$ and the sample $\mathrm{D}$ is followed by the deposition of $4.4 \mathrm{~nm} \quad \mathrm{Al}_{0.37} \mathrm{Ga}_{0.63} \mathrm{As}$ $+50 \mathrm{~nm} \mathrm{GaAs}$ at $510{ }^{\circ} \mathrm{C}$.

For photoluminescence (PL) measurements, the samples were mounted in a closed-cycle He cryostat which provides the temperature of $11 \mathrm{~K}$. PL was excited by an $\mathrm{Ar}^{+}$laser with 
(a)
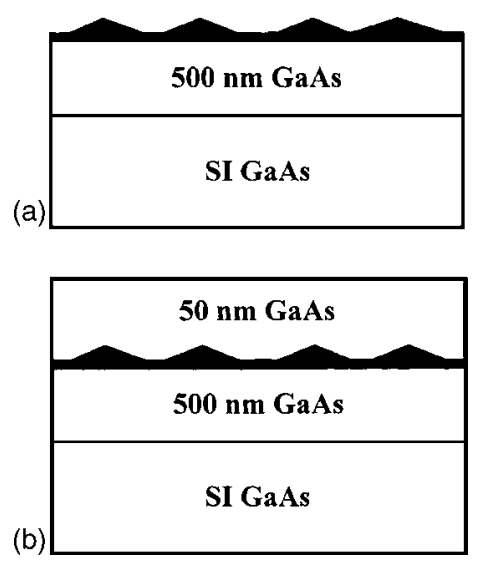

FIG. 1. Schematic structure of (a) sample A for AFM and PL measurements, and (b) samples B for PL measurements.

a $514.5 \mathrm{~nm}$ wavelength and spot size of $300 \mu \mathrm{m}$. Finally, PL through a monochromator was detected by a InGaAs detector whose detection range is below $1.7 \mu \mathrm{m}$.

\section{RESULTS}

Figure 2(a) shows the AFM image of a single InAs QD. The vertical and lateral sizes of QD can be extracted from Fig. 2(b) along [01 $\overline{1}]$ and [011] directions, respectively. The dot height and the base diameter are 7 and $40 \mathrm{~nm}$, respectively. It is clearly seen that the lateral size is much larger than dot height. Therefore, the lateral quantum confinements along $X$ - and $Y$-axes are weak. It is reasonable to assume that

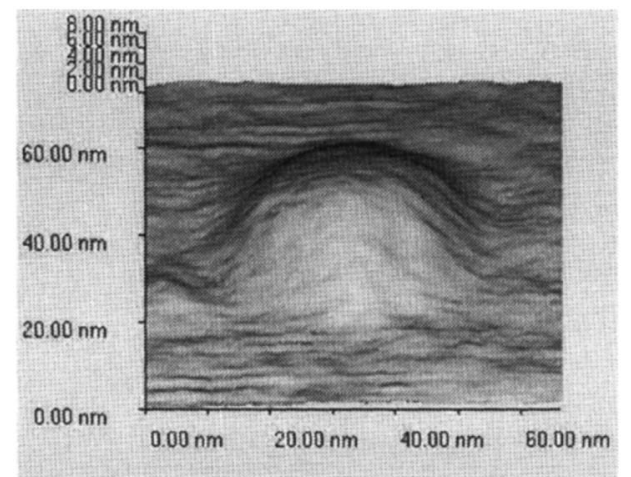

(a)
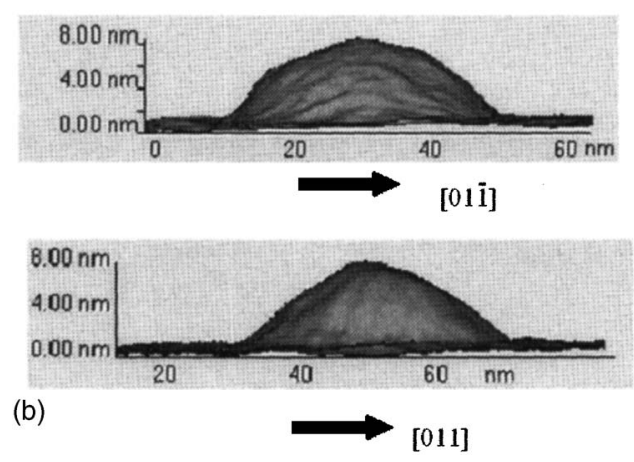

FIG. 2. AFM image of (a) a single InAs QD, and (b) lateral profiles along $[01 \overline{1}]$ and [011] directions.

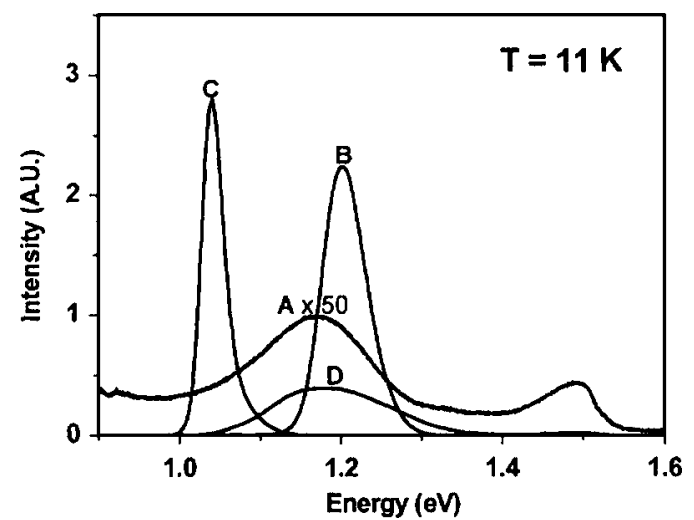

FIG. 3. PL spectra of InAs QD with different cap layers. Sample A: uncapped 3 ML InAs QD; sample B: 3 ML InAs QD capped by $50 \mathrm{~nm}$ GaAs; sample C: 3 ML InAs QD covered by $6 \mathrm{ML} \mathrm{In}_{0.33} \mathrm{Ga}_{0.67} \mathrm{As}$; and sample D: $4.4 \mathrm{~nm}-\mathrm{Al}_{0.37} \mathrm{Ga}_{0.63} \mathrm{As}$, capped QDs.

the energy state of a QD is dominated by the dot height along $Z$-axis, and the weak lateral confinements should be only responsible for the fine structure of PL spectra rather than the peak position. Thus, the InAs/GaAs QD structure can be roughly modeled by one-dimensional quantum well. From the AFM image of a single QD, both the volume and base area of QD can be calculated. That the QD volume divided by the base area gives an average dot height of $3.4 \mathrm{~nm}$ which is defined as the effective quantum well width in the theoretical fittings.

The measured PL spectra are plotted in Fig. 3. The PL peaks of sample A, B, C, and D are 1.163, 1.203, 1.042, and $1.189 \mathrm{eV}$, respectively. It is clear from Fig. 3 that the PL energy difference between uncapped (sample A) and $50 \mathrm{~nm}$-GaAs-capped QDs (sample B) is $40 \mathrm{meV}$, and the difference between 6-ML- $\mathrm{In}_{0.33} \mathrm{Ga}_{0.67} \mathrm{As}$-capped QDs (sample C) and $50 \mathrm{~nm}$-GaAs-capped QDs (sample B) is $161 \mathrm{meV}$. But there is only a little energy difference (14 meV) between GaAs- and $\mathrm{Al}_{0.37} \mathrm{Ga}_{0.63}$ As-capped QDs.

\section{DISCUSSION}

To find out the role the stress plays in determining the energy level of the InAs/GaAs QDs, the experimental data are fitted by theory. Referring to the studies of Li et al., ${ }^{16,17}$ the conduction-band offset of InAs/GaAs heterojunction is assumed to be $70 \%$ of the band gap difference of GaAs and InAs. All the parameters used in the calculations of quantum well are summarized in Table I.

First, the PL peak energy versus band gap of uncapped InAs QDs (sample A) is calculated with fixing the dot height at $3.4 \mathrm{~nm}$. The result is shown in Fig. 4(a). By fitting the experimental result $(1.163 \mathrm{eV})$ of uncapped QDs with the theoretical calculations, the band gap of the uncapped InAs QDs is found to be $0.5 \mathrm{eV}$ which is close to the unstrained case, i.e., $0.418 \mathrm{eV}$. This indicates that the stress in the upper part of the 3.4-nm-thick uncapped InAs QDs is almost relaxed. This relaxation is in good agreement with the observations reported by Lee et al. ${ }^{15}$ 
TABLE I. Parameters used in the theoretical calculation of the energy levels in InAs/GaAs QDs. $m_{e}^{*}$ and $m_{h}^{*}$ are the effective masses of electron and holes in the unit of free electron mass $m_{0} . E_{g}$ is the band gap of GaAs and InAs under different strain conditions.

\begin{tabular}{cccc}
\hline \hline & $E_{g}(\mathrm{eV})$ & $m_{e}^{*}\left(m_{0}\right)$ & $m_{h}^{*}\left(m_{0}\right)$ \\
\hline GaAs & 1.518 & 0.067 & 0.51 \\
Unstrained & 0.418 & 0.023 & 0.41 \\
InAs & & 0.023 & 0.41 \\
$\begin{array}{c}\text { Partially-strained } \\
\text { InAs } \\
\text { Fully-strained }\end{array}$ & $0.418-0.785$ & 0.023 & 0.41 \\
InAs & 0.785 & & \\
\hline \hline
\end{tabular}

Consecutively, the PL energies of GaAs-sandwiched QDs versus dot heights are calculated under different strained conditions, ${ }^{16}$ i.e., the InAs band gap $E_{g}$ varies from 0.418 (unstrained) to 0.785 (fully-strained) $\mathrm{eV}$. The more compressive strain causes the larger InAs band gap. The results are shown in Fig. 4(b). From Fig. 4(b), the slope of unstrained condition as a function of well width is more

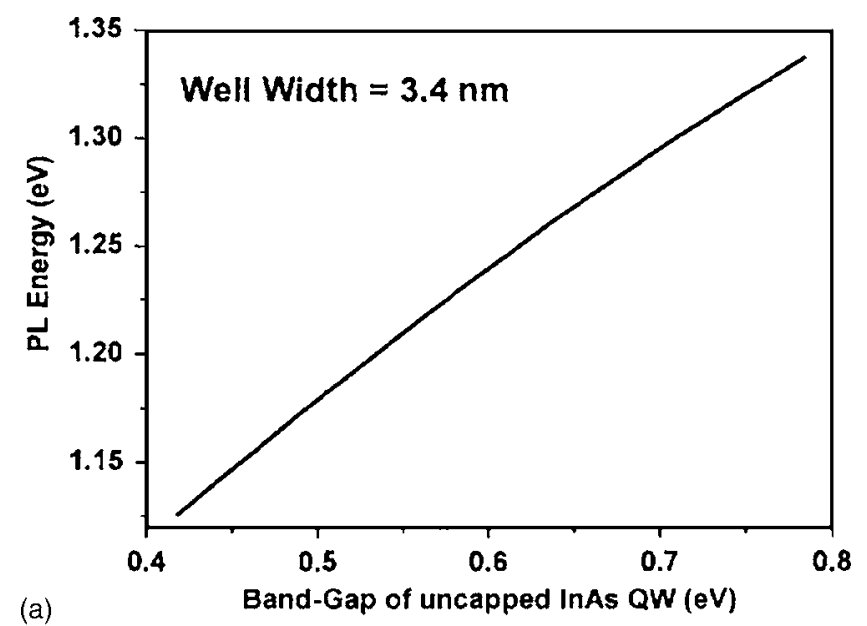

(a)

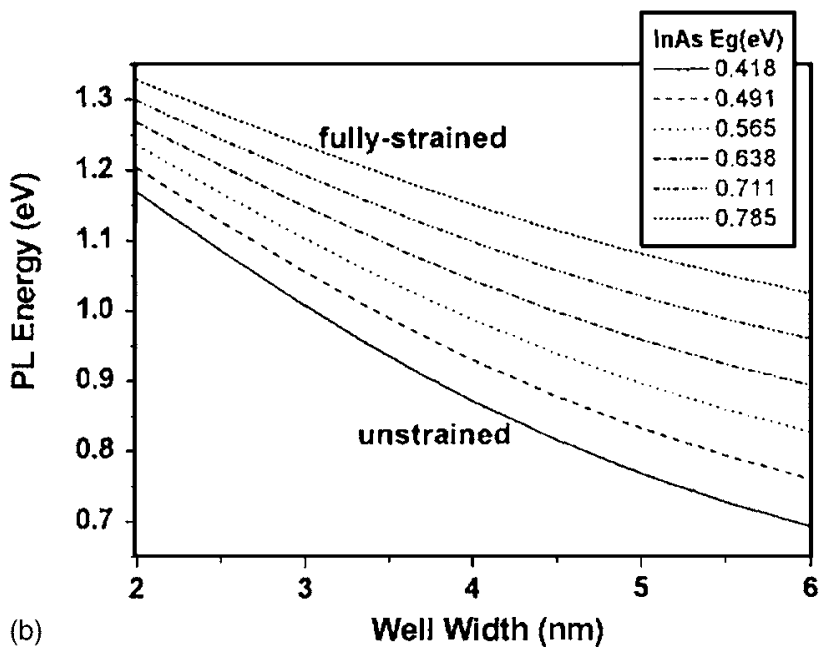

FIG. 4. Theoretical calculations of PL energy as a function of (a) band gap of uncapped InAs QD and (b) GaAs/InAs/GaAs quantum well width with different strain conditions. negative than fully strained one. It tells us that the PL energy is less sensitive to the change of dot height when strained. This is because PL energy is the summation of three energies, i.e., electron energy difference from ground state to conduction band minimum, InAs band gap, and hole energy difference from its ground state to valence band maximum. The well width (dot height) affects electron and hole energy states, while the compressive stress increases InAs band gap. After being compressively strained, the InAs band gap increases and the barrier heights for both electron and hole confined states decrease. The electron (hole) energy separation between ground state and the conduction (valence) band extreme decreases accordingly. Thus, the InAs band gap plays more dominant role in determining the PL energy after strained. It causes less shift of the PL peak energy while the well width increases.

After the InAs QD is capped by the $50 \mathrm{~nm}$ GaAs layer (sample B), the almost-relaxed upper part of the InAs QDs is stressed again. Thus, the dot height (or shape) of the embedded QD becomes slightly different from that of the uncapped one, i.e., $3.4 \mathrm{~nm}$. The cross-sectional transmission electron microscope (TEM) is unable to tell the difference because the strain field of the embedded QD makes the boundary between InAs QDs and GaAs very vague. Usually, other groups take $1 \mathrm{~nm}$ as the finest statistical measuring error. ${ }^{18,19}$ Such error is far beyond the actual variarion of dot height after being capped by GaAs. In other words, the theoretical calculations indicate that the 1-nm change of dot height leads to more than $40-\mathrm{meV}$ shift of PL peak energies which is the difference between sample A and B. A new approach must be adopted. A self-consistent procedure is therefore developed to calculate the dot height. Because the band gap of the uncapped InAs QD is $0.5 \mathrm{eV}$ which is close to the unstrained case, "3.4 nm" is first chosen to be the dot height of fullyrelaxed (unstrained) QD. Since the Poisson ratio of InAs is $0.35,,^{20,21}$ the effective well width of fully-strained InAs QD after capping by GaAs is calculated to be $3.48 \mathrm{~nm}$. This result exhibits a small difference of $0.08 \mathrm{~nm}$ between the uncapped and GaAs-capped QD. For sample B, the PL peak energy is $1.203 \mathrm{eV}$ and the InAs bandgap is calculated to be $0.792 \mathrm{eV}$ using $3.48 \mathrm{~nm}$ as the dot height. However, this value of $0.792 \mathrm{eV}$ is impossible because it exceeds the bandgap of fully-strained InAs QD, i.e., $0.785 \mathrm{eV}$. In other words, this suggests that the GaAs-capped InAs QD is fully-strained and its band gap should be $0.785 \mathrm{eV}$. Using this band gap value of $0.785 \mathrm{eV}$, we can inversely derive the effective well width (dot height) of GaAs-embedded InAs QD (sample B) which is $3.466 \mathrm{~nm}$. By considering the Poisson ratio again, the height of the ideally unstrained InAs QD is extracted to be $3.387 \mathrm{~nm}$ which is smaller than the measured $3.4 \mathrm{~nm}$. Hence, the surface QDs are slightly strained for $0.4 \%$ and its band gap is $0.5 \mathrm{eV}$. In summary, the InAs band gap of 0.418 , 0.5 , and $0.785 \mathrm{eV}$ which correspond to bulk, uncapped, and GaAs-capped InAs QDs indicate the average vertical strains of $0 \%, 0.4 \%$, and $2.3 \%$, respectively. The flow chart of the self-consistent procedure is drawn in Fig. 5. 


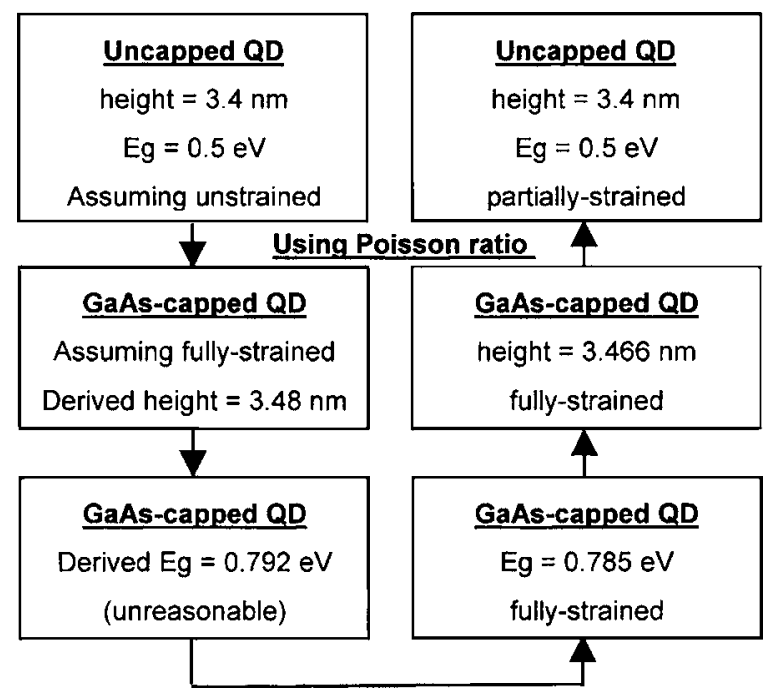

FIG. 5. Flow chart of the self-consistent procedure used in determining the dot height, strain, and resultant band gap of GaAs-capped InAs QDs.

From the discussion above for sample A uncapped InAs $\mathrm{QD}$, the stress coming from the lower GaAs layer results in the vertical strain of $0.4 \%$ and hence a small change of InAs band gap from 0.418 to $0.5 \mathrm{eV}$. But the GaAs-capped InAs QD which is stressed from both upper and lower GaAs has a fully-strained band gap of $0.785 \mathrm{eV}$. This fact indicates that most stresses come from the upper GaAs rather than the lower GaAs buffer layer. It also means that, during the InAs QD growth from 1.7 to $3 \mathrm{ML}$, the strained InAs QD gradually relaxes back to its bulk lattice constant ${ }^{15}$ and finally a very small strain $(0.4 \%)$ remains in the QD.

Additionally, replacing GaAs cap layer with $\mathrm{Al}_{\mathrm{x}} \mathrm{Ga}_{1-x} \mathrm{As}$ in the calculations results in a larger PL peak value, i.e., blueshift which confronts the measured PL spectra, i.e., redshift of $14 \mathrm{meV}$. Joyce et al. $^{22}$ measured the total volume of InAs QDs on (100) GaAs at different growth temperatures and attributed the increase of QD volume to the (In, Ga)As alloying of InAs QDs from lower GaAs material. It was then explained as the InAs/GaAs intermixing due to In segregation. After that, Arzberger et al. ${ }^{23}$ observed and proposed that the InAs/GaAs intermixing caused by In segregation at $T_{S}=530{ }^{\circ} \mathrm{C}$ can be considerably reduced by a thin AlAs capping layer, while the PL spectra of QDs grown at $T_{S}=480{ }^{\circ} \mathrm{C}$ showed a theoretically-predictable blueshift. Other groups also observed by capping InAlAs at $T_{S}=520{ }^{\circ} \mathrm{C}$ and explained with such suppressing process. ${ }^{19,24}$ These results indicate that the substrate temperature determines the ability of In segregation. Higher growth temperature makes the InAs/GaAs intermixing more obvious, while the higher barrier height of capping $\mathrm{Al}(\mathrm{Ga}) \mathrm{As}$ dominates and causes blue shift at $T_{S}=480^{\circ} \mathrm{C}$. Here, another model is proposed to explain these phenomena. Our samples were all grown at $T_{S}=510^{\circ} \mathrm{C}$. The PL spectra show a slightly 14-meV redshift and larger full width half maximum (FWHM) while changing capping layer from GaAs to AlGaAs. As it is known, the melting point of AlGaAs is higher than GaAs. It means that the deposition of AlGaAs can gen- erate more latent heat than that of GaAs while forming solid state on the sample from gas state. This latent heat from AlGaAs loosens the bondings of InAs QDs and result in In/Al intermixing that increases the lattice constant of QDs. The increase of lattice constant will diminish the strain because of the decrease of mismatch between QD and its surrounding material. Once the strain becomes smaller, the strain-induced enlargement of band gap abates, although the permeation of $\mathrm{Al}$ also causes the enlargement of band gap. These two mechanisms compete with each other and result in just a slightly redshift of $14 \mathrm{meV}$. The In/Al intermixing effect also reflects on the broadening of FWHM of PL spectra.

Now, the stress on InAs QDs capped by InGaAs lies between GaAs-covered and cap-free InAs QD. Many researchers therefore claimed that the InGaAs serves as a strainreducing layer. ${ }^{13}$ But our results ${ }^{22}$ and Maximov et al. ${ }^{14}$ have shown that when capping InAs QDs with InGaAs, the InGaAs phase separates into InAs which grows on top of InAs QD and GaAs which grows in the valley between QDs. Base on this phase separation and our simulations, the effective dot height increases from $3.466 \mathrm{~nm}$ to $5.2 \mathrm{~nm}$ when caps 6 ML $\mathrm{In}_{0.33} \mathrm{Ga}_{0.67} \mathrm{As}$ on InAs QDs. It results in the large red shift of PL spectrum from 1.203 to $1.042 \mathrm{eV}$.

\section{CONCLUSIONS}

The effective quantum well model including strain was developed to calculate the energy levels inside the InAs QD and successfully interpreted the PL spectra. The stress mainly comes from the upper GaAs cap layer, not the lower GaAs matrix. The compressive strain makes InAs QD less sensitive to the change of dot height. The slightly 14-meV redshift of changing cap layer from GaAs to AlGaAs results from the In/Al intermixing effect. The large redshift of PL spectrum of InGaAs-capped InAs QD is due to the 1.734$\mathrm{nm}$ increase of dot height, which is caused by the phase separation of InAs and GaAs during growth.

\section{ACKNOWLEDGMENT}

This work is supported by the National Science Council of Republic of China under Contact No. NSC 91-2120-M002-002.

${ }^{1}$ Nien-Tze Yeh, Wei-Sheng Liu, Shu-Han Chen, Pe-Chin Chiu, and Jen-Inn Chyi, Appl. Phys. Lett. 80, 535 (2002).

${ }^{2}$ N. Kirstaedter, N. N. Ledentsov, M. Grundmann, D. Bimberg, V. M. Ustinov, S. S. Ruvimov, M. V. Maximov, P. S. Kop'ev, and Z. I. Alferov, Electron. Lett. 30, 1416 (1994).

${ }^{3}$ D. L. Huffaker, G. Park, Z. Zou, O. B. Shchekin, and D. G. Deppe, Appl. Phys. Lett. 73, 2564 (1999).

${ }^{4}$ K. Mukai, Y. Nakata, K. Otsubo, M. Sugawara, N. Yokoyama, and H. Ishikawa, IEEE Photonics Technol. Lett. 11, 1205 (1999).

${ }^{5}$ A. E. Zhukov, A. R. Kovsh, V. M. Ustinov, Y. M. Shernyakov, S. S. Mikhrin, N. A. Maleev, E. Y. Kondrat'eva, D. A. Livshits, M. V. Maximov, B. V. Volovik, D. A. Bedarev, Y. G. Musikhin, N. N. Ledentsov, P. S. Kop'ev, Z. I. Alferov, and D. Bimberg, IEEE Photonics Technol. Lett. 11, 1345 (1999).

${ }^{6}$ J. Phillips, K. Kamath, and P. Bhattachary, Appl. Phys. Lett. 72, 2020 (1998).

${ }^{7}$ Shiang-Feng Tang, Shih-Yen Lin, and Si-Chen Lee, Appl. Phys. Lett. 78, 
2428 (2001).

${ }^{8}$ H. C. Liu, M. Gao, J. McCaffrey, Z. R. Wasilewski, and S. Fafard, Appl. Phys. Lett. 78, 79 (2001).

${ }^{9}$ Shih-Yen Lin, Yau-Ren Tsai, and Si-Chen Lee, Appl. Phys. Lett. 78, 2784 (2001).

${ }^{10}$ L. Chu, A. Zrenner, G. Böhm, and G. Abstreiter, Appl. Phys. Lett. 75, 3599 (1999).

${ }^{11}$ K. Nishi, H. Saito, S. Sugou, and J.-S. Lee, Appl. Phys. Lett. 74, 1111 (1999).

${ }^{12}$ V. M. Ustinov, N. A. Maleev, A. E. Zhukov, A. R. Kovsh, A. Yu. Egorov, A. V. Lunev, B. V. Volovik, I. L. Krestnikov, Yu. G. Musikhin, N. A. Bert, P. S. Kop'ev, Zh. I. Alferov, N. N. Ledentsov, and D. Bimberg, Appl. Phys. Lett. 74, 2815 (1999).

${ }^{13}$ N.-T. Yeh, T.-E. Nee, J.-I. Chyi, T. M. Hsu, and C. C. Huang, Appl. Phys. Lett. 76, 1567 (2000).

${ }^{14}$ M. V. Maximov, A. F. Tsatsul'nikov, B. V. Volovik, D. S. Sizov, Yu. M. Shernyakov, I. N. Kaiander, A. E. Zhukov, A. R. Kovsh, S. S. Mikhrin, V. M. Ustinov, Zh. I. Alferov, R. Heitz, V. A. Shchukin, N. N. Ledentsov, D. Bimberg, Yu. G. Musikhin, and W. Neumann, Phys. Rev. B 62, 16671 (2000).

${ }^{15}$ H. S. Lee, J. Y. Lee, T. W. Kim, and M. D. Kim, Appl. Phys. Lett. 83,
2256 (2003).

${ }^{16}$ Shu-Shen Li, Jian-Bai Xia, Z. L. Yuan, and Z. Y. Xu, Phys. Rev. B 54, 11575 (1996).

${ }^{17}$ P. D. Wang, N. N. Ledentsov, C. M. Sotomayer Torres, P. S. Kop'ev, and V. M. Ustinov, Appl. Phys. Lett. 64, 1526 (1994).

${ }^{18}$ Yu. I. Mazur, Z. M. Wang, G. J. Salamo, Min Xiao, G. G. Tarasov, Z. Ya. Zhuchenko, W. T. Masselink, and H. Kissel, Appl. Phys. Lett. 83, 1866 (2003).

${ }^{19}$ R. Jia, D. S. Jiang, H. Y. Liu, Y. Q. Wei, B. Xu, and Z. G. Wang, J. Cryst. Growth 234, 354 (2002)

${ }^{20}$ A. Malachias, W. N. Rodrigues, M. V. B. Moreira, S. Kycia, and R. Magalhaes-Paniago, J. Phys. D 36, A249 (2003).

${ }^{21}$ Landolt-Bornstein 1982 Physics of Group IV Elements and III-V Compounds (Springer, Berlin, 1982), Vol. 17a.

${ }^{22}$ P. B. Joyce, T. J. Krzyzewski, G. R. Bell, B. A. Joyce, and T. S. Jones, Phys. Rev. B 58, R15981 (1998).

${ }^{23}$ M. Arzberger, U. Kasberger, G. Bohm, and G. Abstreiter, Appl. Phys. Lett. 75, 3968 (1999).

${ }^{24}$ Z. Y. Zhang, B. Xu, P. Jin, X. Q. Meng, Ch. M. Li, X. L. Ye, and Z. G. Wang, J. Appl. Phys. 92, 511 (2002). 